\title{
Cracked tooth syndrome. Part 2: restorative options for the management of cracked tooth syndrome
}
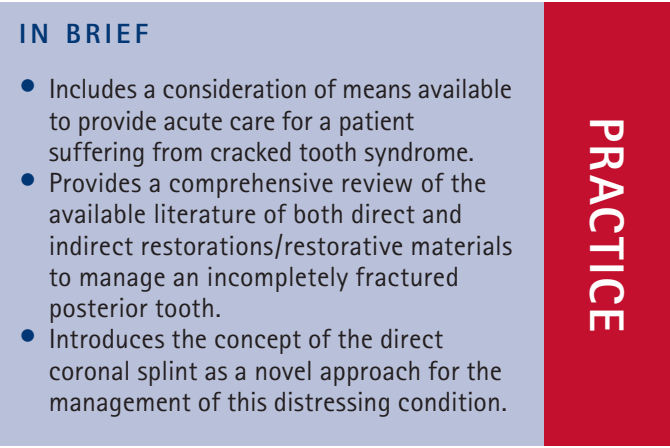

\author{
S. Banerji, ${ }^{1}$ S. B. Mehta ${ }^{2}$ and B. J. Millar ${ }^{3}$ \\ VERIFIABLE CPD PAPER
}

The second of this two part series on 'cracked tooth syndrome' will focus on the available methods for the immediate, intermediate and definitive management of patients affected by this condition. Included in this article is a comprehensive account of the relative merits/drawbacks of various restorative materials and their respective techniques of application for the treatment of symptomatic, incompletely fractured posterior teeth.

\section{PRINCIPLES FOR THE MANAGEMENT OF CRACKED TOOTH SYNDROME}

It is apparent from the first article of this series that the diagnosis of cracked tooth syndrome can pose a major challenge to the dental operator. It would also be fair to state that the management of the condition is by no means always a simple straightforward matter.

While it would appear that there is no universally accepted restorative protocol, it is generally agreed that the aim of restorative therapy is to immobilise the segments of the tooth that move on loading. The latter may be achieved in a limited number of cases simply by the removal of the affected cusp and restoring the defect with an appropriate material, or in the majority of cases by the placement of a restoration that prevents independent movement of the tooth segments on either side of the crack, thereby 'splinting' the tooth together. ${ }^{1}$

The process of splinting should minimise flexure of the compromised cusp, therefore not only aiming to alleviate the symptoms of pain on biting but also prevent further

1.Senior Clinical Teacher: ${ }^{2}$ General Dental Practitioner and Clinical Teacher, ${ }^{3 *}$ Professor and Consultant in Restorative Dentistry, Department of Primary Dental Care, King's College London Dental Institute, Bessemer Road, London, SE5 9RW

${ }^{*}$ Correspondence to: Professor B. J. Millar

Email: brian.millar@kcl.ac.uk

\section{Refereed Paper}

Accepted 25 March 2010

DOI: $10.1038 /$ sj.bdj.2010.496

${ }^{\circledR}$ British Dental Journal 2010; 208: 503-514 propagation of the crack and reduce the ingress of bacterial microorganisms into the dental pulp. ${ }^{2}$

Historically a plethora of different 'forms' of dental restorations have been advocated to 'splint teeth' affected by incomplete fractures. This paper will divide these into four broad categories. Hence:

- Immediate

- Direct restorations placed intracoronally without cuspal coverage

- Direct restorations, which provide cuspal coverage

- Indirect restorations placed intracoronally without any cuspal support and indirect restorations which provide cuspal coverage (onlays and full coverage restorations).

The aim of this article is to review the available literature on the above restorations when used to treat cases of cracked cusp syndrome, with emphasis placed on the efficacy, efficiency, economic viability and biological cost of each of the above.

\section{IMMEDIATE THERAPY OF CTS}

It is generally accepted that the more rapidly a tooth with a crack is treated, the easier it will be to avoid irreversible damage. Table 1 provides a list of the possible immediate treatment options. As an 'immediate' or remedial approach a number of authors have advocated the undertaking of occlusal adjustments upon affected teeth, to reduce the stress on the tooth, so as to not only prevent further

\begin{tabular}{|c|c|}
\hline \multicolumn{2}{|c|}{$\begin{array}{l}\text { Table } 1 \text { Immediate treatment options } \\
\text { for CTS } \\
\text { Occlusal adjustment - destructive, short term }\end{array}$} \\
\hline \multicolumn{2}{|c|}{ Remove segment - highly destructive } \\
\hline \multicolumn{2}{|c|}{ Immobilisation of segment: } \\
\hline Copper ring & $\begin{array}{l}\text { difficult and } \\
\text { periodontally damaging }\end{array}$ \\
\hline $\begin{array}{l}\text { Orthodontic } \\
\text { band }\end{array}$ & $\begin{array}{l}\text { time consuming, } \\
\text { possibly not available }\end{array}$ \\
\hline $\begin{array}{l}\text { Temporary } \\
\text { crown }\end{array}$ & $\begin{array}{l}\text { highly destructive, } \\
\text { time consuming }\end{array}$ \\
\hline $\begin{array}{l}\text { Direct } \\
\text { composite splint }\end{array}$ & $\begin{array}{l}\text { quick, low cost, readily } \\
\text { available and non-invasive }\end{array}$ \\
\hline
\end{tabular}

crack propagation but also relieve the associated symptoms. ${ }^{3}$

However, it has been argued that simply grinding the tooth out of occlusion is likely to be only of limited benefit as the tooth may still be critically stressed by a food bolus to such an extent that the process of flexure will still continue on loading. ${ }^{4}$ Furthermore, occlusal adjustment may not only involve the removal of healthy sound tooth tissue, but when undertaken without analysing the effects on the residual dentition may also lead to unwanted occlusal interferences elsewhere in the dental arch.

Fox et $a l .{ }^{5}$ have recommended that following a positive diagnosis of a cracked tooth where a pre-existing restoration is present, it should be removed to assess the full extent of the fracture. Often during this process the affected cusp may 'splinter off' and the remaining defect managed accordingly. 
Where there has been no splintering off the affected portion of the tooth, immediate immobilisation by means of an 'immediate extra-coronal circumferential splint' may be applied. The latter may take the form of a copper ring or a stainless steel orthodontic band, both of which should be tight fitting, contoured to not interfere with the occlusion and cemented using zinc-polycarboxylate cements. ${ }^{6}$

It is generally agreed however that stainless steel orthodontic bands are the preferred option, as they can be contoured more effectively so as to inflict less irritation/damage to the gingival/periodontal tissues. Upon review, following a period of two to four weeks after the application of the immediate splint, the absence of pain has been described to indicate not only a correct diagnosis but also successful immobilisation. ${ }^{7}$ Where symptoms of thermal sensitivity persist, endodontic therapy may need to be considered. ${ }^{7}$ In such cases, the splint may remain in situ until the completion of root canal therapy and the eventual application of the definitive restoration.

While the placement of copper rings and stainless steel bands have been suggested to be a clinically effective, minimally invasive and a cost effective immediate treatment modality for teeth affected by cracked tooth syndrome, ${ }^{7}$ in the opinion of the authors, the technique of placing the latter may be time consuming and technically challenging. Furthermore, with the advent of adhesive dentistry, many dental operators may not have acquired the technical experience needed with the placement of copper rings; indeed, copper rings may not always be readily available in the modern general dental practice. Likewise, many general dental practitioners may not have the technical expertise or ready access to stainless steel orthodontic bands. Discomfort associated with the application of copper rings and their tolerance may be other complicating factors. In addition patients may object to the aesthetics where the band is visible.

The preparation and placement of full coverage acrylic provisional crowns has been described by Gutherie et al. ${ }^{8}$ as a predictable, 'immediate splinting' option for the management of incompletely fractured teeth. Preformed crowns of differing varieties may also be applied in a similar way. The placement of such restorations while effective are however, time consuming, biologically invasive and costly. Furthermore, rarely in the opinion of the authors is it possible to prepare a tooth to receive a full coverage provisional crown when a patient first presents with CTS on the same appointment. It is worthwhile noting however that any delay in instituting immediate therapy may lead to further progression of the fracture and in the worst scenario culminate in tooth loss as well.

A novel concept which has yet to be fully supported by a clinical trial is the placement of bonded composite resin directly over the surface of the tooth to splint across the fracture and immobilise the segments (Fig. 1). The splinting method is used by all the authors and is referred to as a 'direct composite splint' (DCS). It can be used with minimal tooth reduction of the functional areas of the occlusal surface but the authors now prefer to use it as a non-invasive splint with no tooth reduction, creating a flat splint in a supra-occlusal position. Resin composite is applied to a thickness of 1.0-1.5 mm over the occlusal surface of the affected tooth, and finished just beyond the external line angles of the affected tooth. The restoration should be contoured to display the absence of occlusal contacts in either lateral or protrusive mandibular movements (which may require the addition of resin composite to guiding teeth).

The above approach of placing a restoration in supra-occlusion follows the principles of the well documented Dahl concept $\mathrm{t}^{9-11}$ whereby it would be expected, in cases other than those with limited eruptive potential, that through the combined process of dento-alveolar segment intrusion and extrusion respectively, occlusal contacts would be re-established after a suitable period of time.

A DCS has the potential, in theory, to be used as both an immediate and intermediate restorative option. It has the potential of being biologically conservative, aesthetic, efficient to apply, reversible and an inexpensive restorative modality, which also offers the potential to offer immediate pain relief and assist in diagnosis (particularly where there may be doubt). However, careful case selection and the attainment of patient consent are very important factors to consider. A detailed
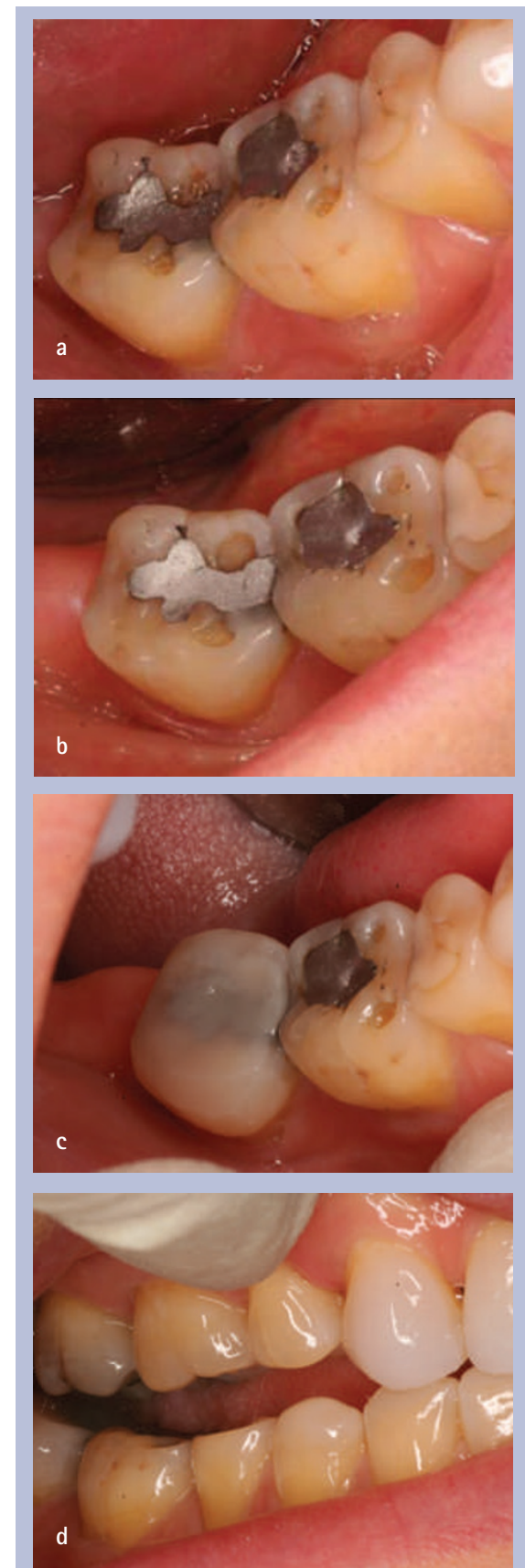

Fig. 1 A tooth with amalgam restoration and a mesial crack (a). Air abrasion was used to augment micro-mechanical retention (b) and a direct composite splint was placed in supra-occlusion (c) without any tooth preparation which is designated as an immediate option for the management of an incompletely fractured, symptomatic lower right second molar tooth. (d) demonstrates the importance of ensuring that the splinted tooth is free of occlusal contact on mandibular lateral excursive movement

literature review has been published by Poyser et al. ${ }^{12}$ documenting the principles of the Dahl concept.

A DCS may also be used before the application of an adhesive onlay (Fig. 2), where the desired inter-occlusal space for 
the definitive restoration may be accomplished by the process of controlled intrusion and extrusion of the dento-alveolar segments. The application of DCS restorations does however require considerable further research to validate its application in every day dental practice.

\section{THE USE OF DIRECT/PLASTIC INTRA-CORONAL RESTORATIONS (WITHOUT CUSPAL COVERAGE) TO TREAT CASES OF CTS}

\section{Dental amalgam (conventional/bonded)}

Dental amalgam, composite resin and glass-ionomer cements are among three of the most commonly used plastic, direct restorative materials in contemporary dental practice. In principle, their use intracoronally in the management of cases of CTS (in the absence of any splintering) is based on their ability to attach themselves to the areas of tooth tissue located either side of the fracture and thereby prevent independent movement of either fragment upon loading. In doing so, it would be hoped that these materials would restore the fracture strength of the tooth equivalent to that of a sound caries free tooth. ${ }^{13}$

Longitudinal studies on the performance of extensive amalgam restorations have confirmed amalgam's success as a direct, plastic restorative material. ${ }^{14,15}$ However, the fact that dental amalgam lacks intrinsic adhesive potential would infer that it would have little effect on 'binding' fractures or restoring fracture resistance unto teeth affected by CTS (without the need of auxiliary retentive aids).

It has been shown that fresh dental amalgam can be bonded to etched enamel and dentine with an 'adhesive liner, ${ }^{16}$ hence the concept of the 'bonded amalgam restoration'. Two case reports have been published involving the successful application of bonded amalgam restorations for patients presenting with CTS. The first, a single clinical case involving the use of 'Amalgabond'; ${ }^{17}$ the adhesive agent present in Amalgabond contains 4-META (4-methacryloxylethyl-trimellitate anhydride). 4-META has been shown to have the capacity to bind with collagen fibrils present in the organic component of dentine and to metallic ions. ${ }^{18}$ The second case report included four patients
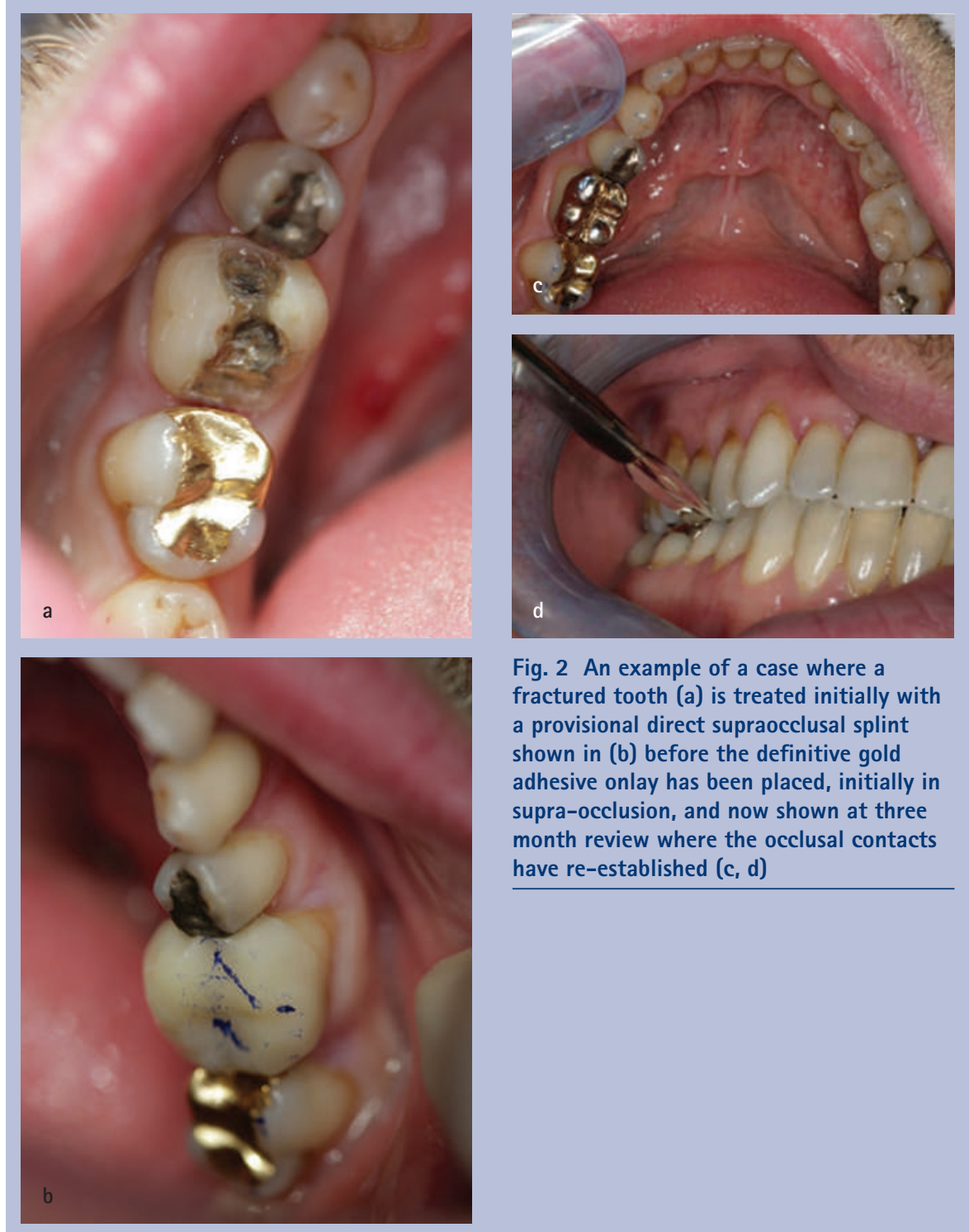

Fig. 2 An example of a case where a fractured tooth (a) is treated initially with a provisional direct supraocclusal splint shown in (b) before the definitive gold adhesive onlay has been placed, initially in supra-occlusion, and now shown at three month review where the occlusal contacts have re-established $(c, d)$

suffering from CTS that had been managed by the application of bonded amalgam restorations, bonded using Panavia EX (Kuraray, Japan). ${ }^{19}$

Panavia EX is a chemically active resin that bonds to both enamel and metal. ${ }^{20}$ Bonds strengths of 8.8-14.2 $\mathrm{MPa}$ have been reported to develop between etched enamel and Panavia EX, ${ }^{19}$ versus that of 18-20 MPa between composite resin and etched enamel.

In vitro studies have reported the fracture strength of molar teeth containing bonded amalgam MOD restorations to be significantly higher than when compared to conventional MOD amalgam restorations. ${ }^{21,22}$

\section{Directly bonded resin composites}

Composite resin offers an aesthetic alternative to silver amalgam for the restoration of posterior teeth. Figures 3-6 illustrate examples of direct composite resin used to restore fractured teeth. In Figure 3 conventional tooth reduction was carried out before composite placement. Figures 4 and 5 show cases where only minimal reduction was carried out and the composite was placed at the original vertical dimension. Figure 6 shows a similar case with no occlusal reduction where the composite resin was placed in supra-occlusion.

The results of in vitro studies regarding the ability of resin composite to restore cuspal stiffness are conflicting in outcome; while some have reported little marked improvement ${ }^{23}$ others have shown that the placement of composite resin restorations has the potential to restore fracture strength, and in some cases to a level superior to that of a healthy, un-prepared tooth. ${ }^{24}$

Opdam et al. ${ }^{25}$ investigated the short term clinical efficacy of bonded (direct) composite 


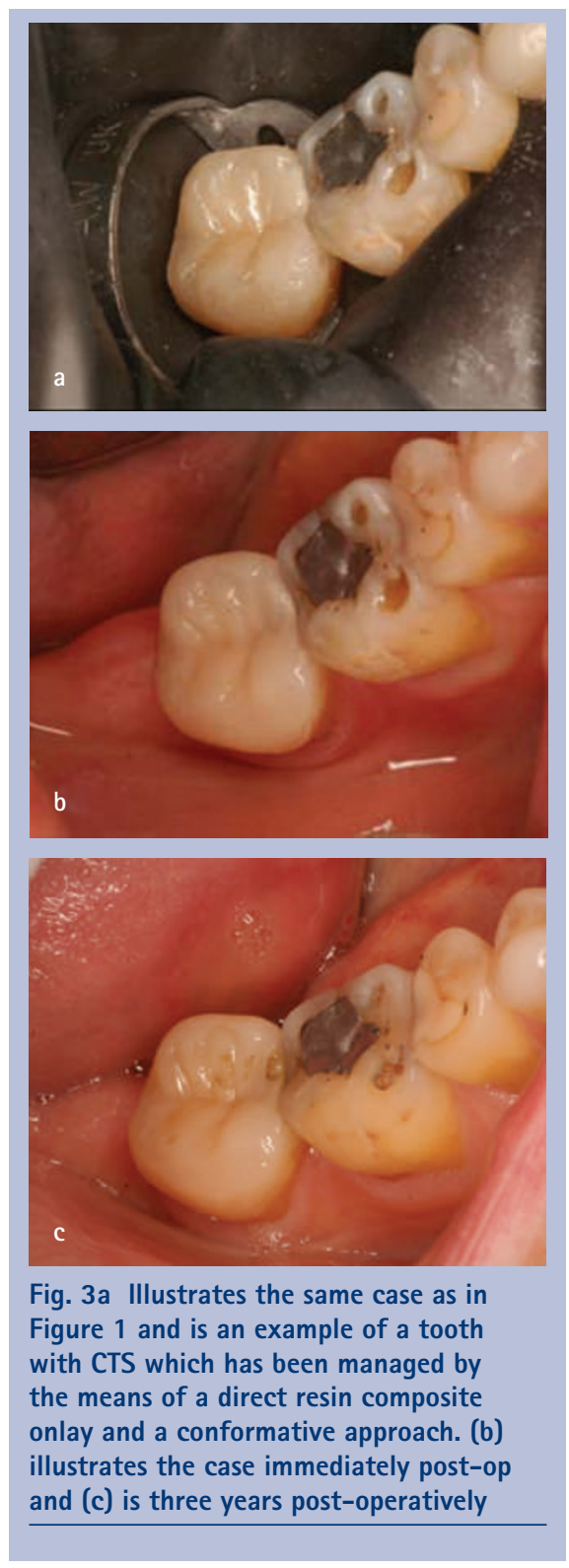

resin when placed intra-coronally for the treatment of 21 painful, cracked teeth. The authors concluded that direct composite resin restorations without cuspal coverage can be effective in treating cases of CTS (75\% of cases reported alleviation of symptoms when verbally questioned). Opdam et al. ${ }^{26}$ have also published a report in 2008, documenting the results of a seven year evaluation of the efficacy of direct composite resin when used to restore painful cracked teeth (previously containing amalgam restorations). The results of the latter study displayed an annual failure rate of $6 \%$.

It has been postulated that in the absence of cuspal coverage, repeated loading of the restoration or residual tooth tissue may stress the 'adhesive layer' and ultimately lead to its breakdown, with the concomitant failure of the ability of the restoration
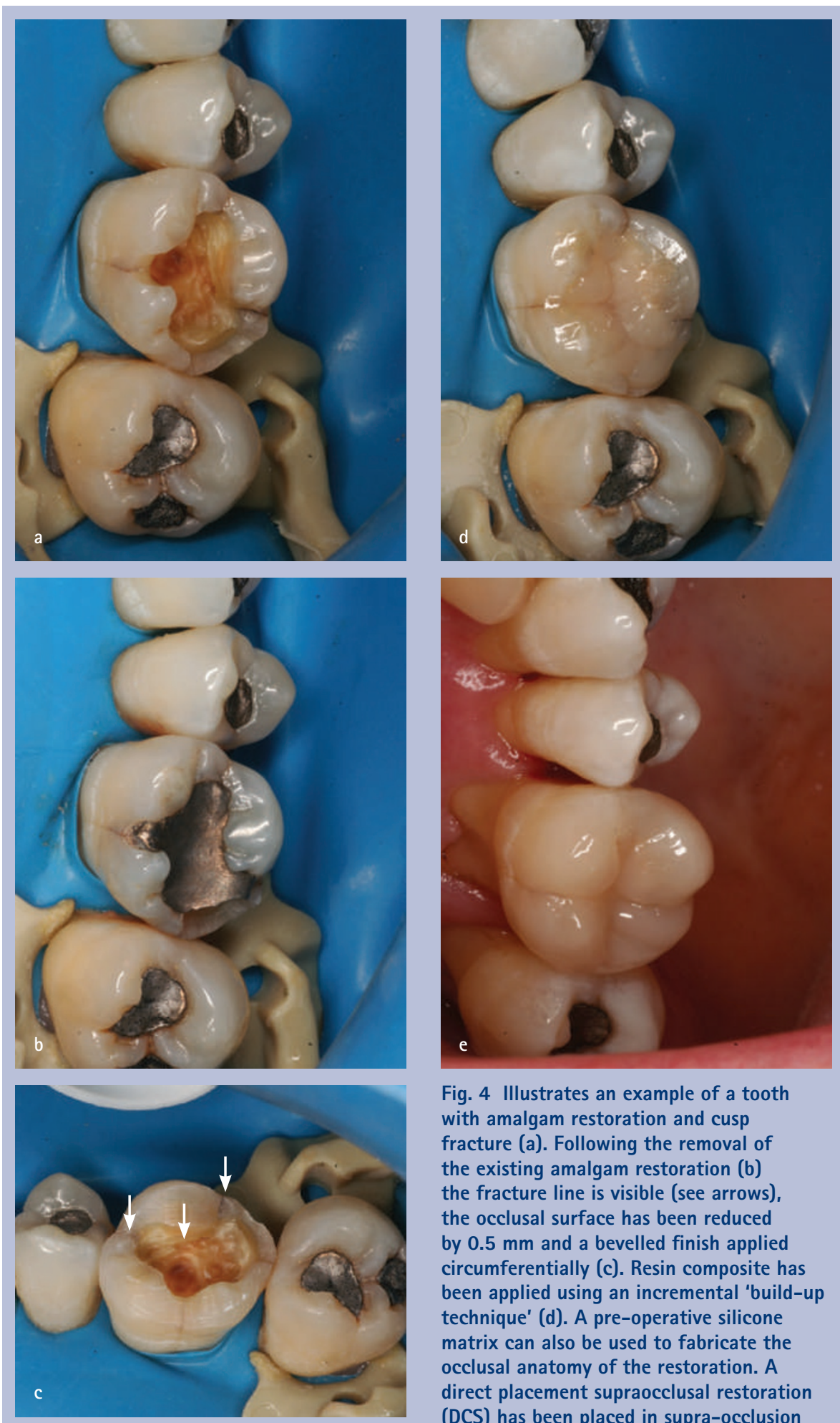

Fig. 4 Illustrates an example of a tooth with amalgam restoration and cusp fracture (a). Following the removal of the existing amalgam restoration (b) the fracture line is visible (see arrows), the occlusal surface has been reduced by $0.5 \mathrm{~mm}$ and a bevelled finish applied circumferentially (c). Resin composite has been applied using an incremental 'build-up technique' (d). A pre-operative silicone matrix can also be used to fabricate the occlusal anatomy of the restoration. A direct placement supraocclusal restoration (DCS) has been placed in supra-occlusion with minimal tooth preparation, which may be retained as an intermediate restoration in the short to medium term (e)

to effectively splint the tooth. ${ }^{26}$ The latter effect may be exaggerated in cases of CTS by two factors; firstly, CTS is often seem among patients who display parafunctional grinding habits who would be more likely to apply higher loads to the restored tooth, and secondly due to the presence of a pre-existing fracture, cuspal flexibility would have already been increased when compared to a healthy un-fractured, sound tooth, placing further greater strain on a progressively weakening adhesive layer. 
It has been reported that resin bonded composites (and bonded amalgam restorations) are less effective in restoring fracture resistance where cavities are greater than half of the intercuspal width (without the use of cuspal coverage)..$^{23}$ The incremental application of composite resin to a cavity (versus bulk placement) also has a significant impact on improving the ultimate fracture resistance of a restored tooth. ${ }^{27}$

Polymerisation shrinkage is a factor commonly cited as an important cause for the higher reported failure rate associated with large composite resin restorations. ${ }^{28}$ Shrinkage may also cause the flexion of the cusps towards the bulk of the material which may further accentuate the fracture, and also induce stresses in the material and the adhesive layer, which would in turn lead to the failure of the ability of the material to immobilise the crack.

The feature of polymerisation shrinkage may, however, be vital to the success reported for direct resin composite onlays, as it may help to splint the crack by effectively embracing the cusps together, as polymerisation shrinkage will cause the material to shrink towards the centre of the restoration and add up to the bonded surface. ${ }^{29}$

Other factors which should also be taken into consideration when considering the application of resin composite in posterior teeth include, the risks of restoration bulk fracture and accelerated wear. The attainment of high quality proximal contact points and occlusal anatomical form may also be a concern when using direct composite.

\section{Glass ionomer cements (GICs)}

The role of glass ionomer cements (GICs) in the management of cases of incompletely fractured posterior teeth to the knowledge of the authors has not been extensively documented. It has been suggested that while GICs have the capacity to increase tooth stability among teeth with class II posterior cavities, they offer a lower bond strength to both enamel and dentine when compared to resin composite. ${ }^{30}$ Furthermore, due to their inferior wear and fatigue properties (compared to silver amalgam and composite resin), they are not the first choice of material for application in stress bearing posterior cavities. ${ }^{30}$

Silver reinforced glass ionomer cements
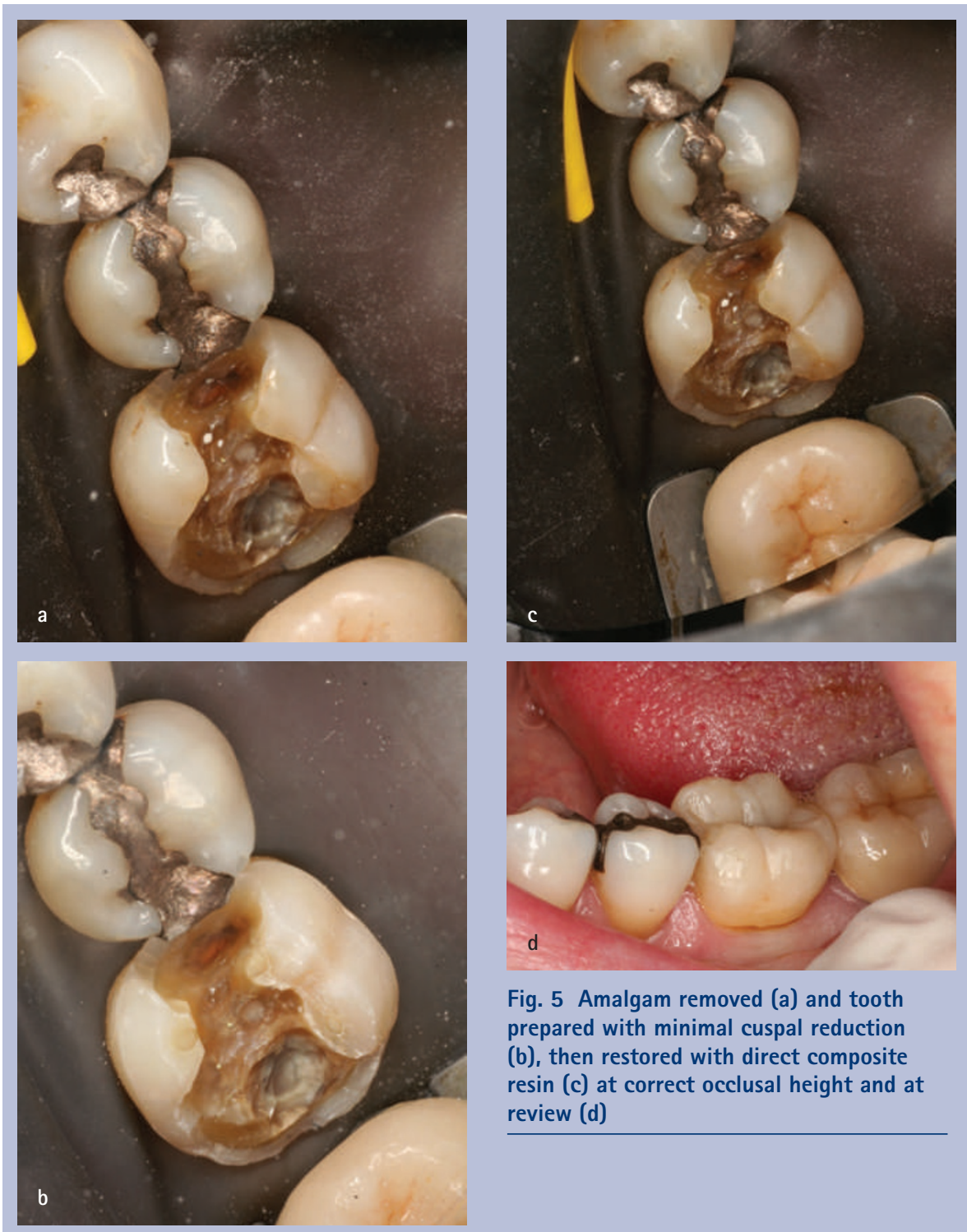

Fig. 5 Amalgam removed (a) and tooth prepared with minimal cuspal reduction (b), then restored with direct composite resin (c) at correct occlusal height and at review (d)

(cermets), however, have been reported to restore prepared teeth to a strength equivalent to that of sound teeth or those restored with resin composite. ${ }^{31}$ The poor aesthetics associated with cermets is a concerning factor.

GICs may have a role to play in the management of cases of CTS as base materials under extensive direct composite resin fillings thereby reducing the volume of resin required and the associated levels of polymerisation shrinkage. ${ }^{32}$

In summary, direct plastic restoratives (bonded amalgam and resin composite restorations), when applied without cuspal coverage to manage cases of cracked tooth syndrome, have the potential to provide a conservative, cost effective and time efficient option to the use of indirect alternatives. While it has been proposed that it takes 2.5 times longer to place and complete a complex composite resin restoration compared to similar amalgam restorations, ${ }^{33}$ it still takes less time than to complete an indirect restoration. All too often, where a patient has had to return following a diagnosis of an incomplete fracture for the preparation of an indirect restoration, the fracture may have progressed to the pulp or culminated in the loss of an extensive amount of the tooth.

According to a leading authority, cracked teeth should be considered to be at an increased risk of pulpal complications (in particular, where there has been thermal sensitivity); ${ }^{26}$ the preparation of such teeth to receive indirect restorations would not only result in the loss of more sound tooth tissue but necessitate the use of a provisional restoration. Both the latter factors will increase the risk of pulpal complications. 


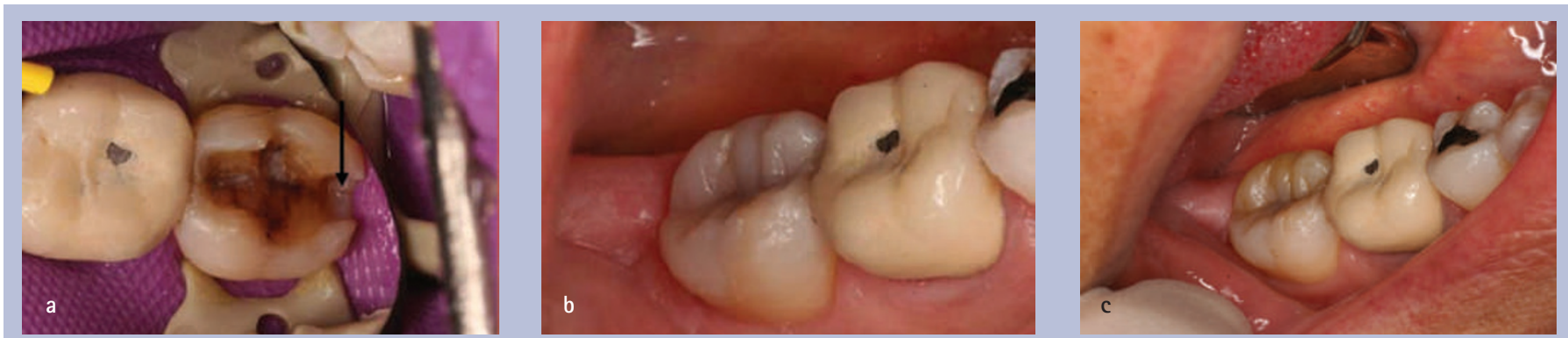

Fig. 6 A cavity showing amalgam removed and crack visible (see arrow) on distal margin (a). The tooth was restored without cusp reduction and directly placed composite as a supraocclusal composite splint. (b) shows immediate post-op view and (c) is three years post op

It has been reported by Gutmann et al. ${ }^{34}$ that approximately one fifth of all teeth diagnosed with cracked tooth syndrome will eventually require endodontic therapy; furthermore the prognosis of endodontically treated, cracked teeth appears to be relatively poor. A study by Tan et $a l .{ }^{35}$ reported a failure rate of $14.5 \%$ for cracked, root filled teeth after a relatively short evaluation period of just two years.

Caution does need to be exercised when considering the use of either direct bonded amalgams or resin composites for cavities which are greater than half the intercuspal width in cases of CTS, ${ }^{23}$ and perhaps among patients who display signs of bruxism.

\section{DIRECT RESTORATIONS \\ WITH CUSPAL COVERAGE; DIRECT OVERLAYS/ONLAYS}

\section{Direct amalgam overlays (conventional/bonded)}

Hood et al. in $1991^{36}$ reported that teeth restored with amalgam overlays had 'fracture energies equivalent to that of intact teeth'. Accordingly, Homewood ${ }^{37}$ has advocated the overlaying of the cusps of teeth affected by CTS as a primary mode of treatment for such cases. The latter undertook an analysis to assess the performance of conventional amalgam overlays for the management of cases with CTS, and reported a very high success rate (in excess of 93\%). ${ }^{37}$

Davis et al. ${ }^{38}$ have also published the results of a clinical study to compare the efficacy of conventional pin retained complex amalgam fillings versus bonded amalgam restorations. A similarly high proportion of cases displayed resolution of symptoms as reported by Homewood. ${ }^{37}$ Interestingly, the results of the study by Davies et $a l .{ }^{38}$ showed a further reduction in cold sensitivity was observed to occur in the group treated by the means of bonded amalgam overlays following a two week post-operative period (while the baseline levels for thermal sensitivity remained unchanged for the non-bonded group). The latter observation may be accounted for by the ability of the resin liner to seal patent dentinal tubules, but also by the fact that the non-bonded group required $4 \mathrm{~mm}$ of tooth reduction of the involved cusp (to accommodate the pin[s]) as opposed to 2 $\mathrm{mm}$ for the bonded group, which may have made a difference in the pulpal response to cold.

The results of both studies ${ }^{37,38}$ have indicated a possible role for amalgam overlays in the management of incomplete fractures in posterior teeth (at least in the short term), particularly where a bonded amalgam restoration is applied.

\section{Directly bonded resin composite onlays}

According to the results of an in vitro study, where premolar teeth were assessed for fracture resistance where composite resin restorations had been placed both with and without cuspal coverage, cases with cuspal coverage displayed a significantly higher fracture resistance. ${ }^{39}$

The above was reflected in two clinical studies by Opdam et al. ${ }^{25,26}$ In both studies, following the removal of the existing amalgam restoration, the cusps of affected teeth (as opposed to the entire occlusal surface) were reduced by $0.5-1.0 \mathrm{~mm}$ coronally from the onset of the micro-crack and the resulting cavity outline finished with a bevelled margin (before the application of direct composite resin).

In the more recent of the two studies, cases that were included were evaluated for a period of six to seven years. ${ }^{26}$ At the end of the observation period, a survival rate of 100\% was elucidated; all 21 cases reported the elimination of symptoms of thermal sensitivity and pain on biting when asked verbally. Similar results were published by Homewood. ${ }^{37}$

The application of resin composite over the affected cusp in cases of CTS appears to reduce the stress on the weakened cusp, possibly by a combination of factors such as composite resin itself having some level of 'shock absorbing effect' ${ }^{25}$ by increasing cuspal stiffness and by re-distributing occlusal loads away from the crack towards the axial walls and down the long axis of the tooth. A reduction in the height of the affected cusp may also reduce its level of flexion upon loading, which may also help in the management of symptoms but also reduce the stress on the adhesive layer, and thereby enhance the longevity of the restoration.

Figure 3 shows an example of a tooth with CTS, which has been managed by the means of a direct resin composite onlay and a conformative approach. Figure $3 c$ has been taken of the same restoration, three years post-operatively.

A success rate of $72.7 \%$ has been reported for teeth restored by means of direct composite resin inlays/onlays (over a period of 11 years); fracture, occlusal wear and secondary caries were the prime causes of failure. ${ }^{28}$ However, Bartlett et al. ${ }^{40}$ have contraindicated the use of direct (or indirect) composite resin for the treatment of worn posterior teeth.

In summary, it would appear that the use of plastic materials (bonded amalgams and bonded resin composite) when placed over the affected cusp in cases of cracked tooth syndrome seems not only to be time and cost effective but also clinically successful in the alleviation of associated 
symptoms. Further studies are however required to assess the long term efficacy of bonded amalgams in cases of CTS. Pulpal involvement does not appear to be a major issue either where direct overlays have been applied to manage CTS, which is a concern where indirect methods are used. Should endodontic therapy, however, be required where a plastic direct material has been applied, the cost implications are minimal and access to the pulp would be relatively straightforward, as opposed to in the presence of an indirect casting, which may need to be re-fabricated following the completion of root canal therapy (while the direct restoration may be readily repaired).

The prime concern with the application of direct overlays is the level of tooth reduction, the need for considerable operator skill and the longevity of these restorations, particularly among bruxists.

\section{INDIRECT RESTORATIONS}

\section{Inlay restorations \\ (conventional and adhesive)}

Conventional intra-coronal inlay restorations utilise the concept of 'wedge retention', which has the potential of exerting an outward pressure on the tooth. ${ }^{41}$ Pressure may be exerted not only upon trial of the restoration (pre-cementation) but also during its functional lifespan, where cyclical occlusal loads are applied. Consequently, conventional inlays have absolutely no role in the treatment of cases of CTS.

With an increase in the demand by dental patients for more aesthetic, predictable posterior tooth restoratives, ceramic inlays and indirect composite inlays have become commonplace in contemporary dental practice as an alternative to direct composite resin.

To the authors' knowledge, to date there have been no reports published documenting the efficacy of adhesive aesthetic (resin or ceramic inlays) for the treatment of CTS. An in vitro analysis has shown that bonded MOD ceramic (Cerec) inlays and laboratory fabricated indirect resin bonded composite inlays have the potential to increase the fracture strength of human molar teeth with wide cavities to values of similar strength to that of sound, healthy teeth. ${ }^{42}$

The use of adhesive inlays may only have a limited role in the management of painful, cracked teeth. Their application is time consuming, sometimes necessitating the use of a provisional inlay (which may further accentuate the crack) and require the removal of healthy hard tissue undercuts, which is biologically invasive. Furthermore, a five year study by Wassell et al.,43 when comparing the failure rates, wear rates and other aspects of clinical performance of direct composite inlays versus conventional composite resin restorations placed incrementally, revealed no significant statistical difference between the two forms of dental restorations.

\section{INDIRECT RESTORATIONS WITH CUSPAL COVERAGE (ONLAYS AND FULL/PARTIAL COVERAGE CROWNS)}

While direct restorations placed intracoronally (without cuspal coverage) have a role in the management of cracked painful teeth, consensus opinion is that for wider cavities in particular, there is a need for cuspal coverage to further protect and strengthen the residual tooth structure. ${ }^{23}$ The fabrication of direct restorations which provide cuspal coverage is considerably demanding of operator skill; the longevity of direct plastic restorative materials may also be a concern. The remainder of this article will focus on indirect restorations which provide cuspal coverage for the treatment of CTS.

\section{ONLAY RESTORATIONS}

\section{Conventional/adhesive metal onlays}

The procedure for the preparation of a tooth to receive a conventional cusp covered gold onlay has been meticulously described and has been advocated for the conservative management of damaged occlusal surfaces of posterior teeth. ${ }^{44}$ While historically they have been widely used for the management of CTS, there appears to be little clinical data to document their effectiveness in this application.

The advent of newer generation dentine bonding systems and the availability of low viscosity chemically active cements to bond tooth structure to either metals, ceramic or composite resin, has paved the way for restorations which may be used to treat dental conditions in a very 'minimally invasive manner. ${ }^{45}$ The adhesive metal onlay is an example of a minimally invasive, indirect restoration that has been used to treat cases of cracked tooth syndrome' ${ }^{46,47}$ Examples are illustrated in Figures 2 and 7.

$\mathrm{Yap}^{47}$ has presented a case report documenting the application of a resin bonded (adhesive) metal onlay for the management of a patient presenting with CTS, involving the placement a cast cobalt chromium alloy (Degussa, Germany). The preparation involved the reduction of the occlusal surface by $1.0 \mathrm{~mm}$ over the functional cusps and $0.7 \mathrm{~mm}$ over the non-functional cusps; a shoulder design finish line was applied circumferentially, $1.2 \mathrm{~mm}$ beneath the prepared occlusal surface. The restoration was reported to be in place for a period of at least one year and the tooth described to be asymptomatic. The use of cobalt-chromium alloys on account of their extreme rigidity permits an ultraconservative preparation.

The successful application of resin bonded alumina abraded type III cast gold alloy onlays (luted with Panavia EX, Kuraray, Japan) to treat six cases of CTS has also been described. ${ }^{46}$ In the latter study, all affected teeth were prepared to accommodate metal to the thickness of 1.5-1.0 $\mathrm{mm}$ and restorative margins were finished on enamel tissue, with a 'small chamfer' placed 1-2 mm circumferentially below the prepared occlusal surface. Restorations were evaluated for a mean service period of 48 months; no failures were noted.

Figure 7 shows an incompletely fractured symptomatic lower molar tooth which has been restored by the application of a gold adhesive onlay. The tooth has been prepared to receive a cast type III gold adhesive onlay; the occlusal surface has been reduced by 1.0-1.5 mm and a chamfer margin applied circumferentially. This cast gold overlay was conformative and fitted to the existing vertical dimension.

The use of type III or type IV cast gold alloys offer the merits of favourable wear characteristics, ease of casting and finishing, high corrosion resistance and ease of burnishing. ${ }^{46}$ The formation of an oxide layer on the fit surface of gold alloy adhesive onlays by heat treatment at $650^{\circ} \mathrm{C}$ for ten minutes or tin plating is thought to increase adhesion with the chemically active resin lutes. ${ }^{48}$

The adhesive onlay restoration quite clearly 

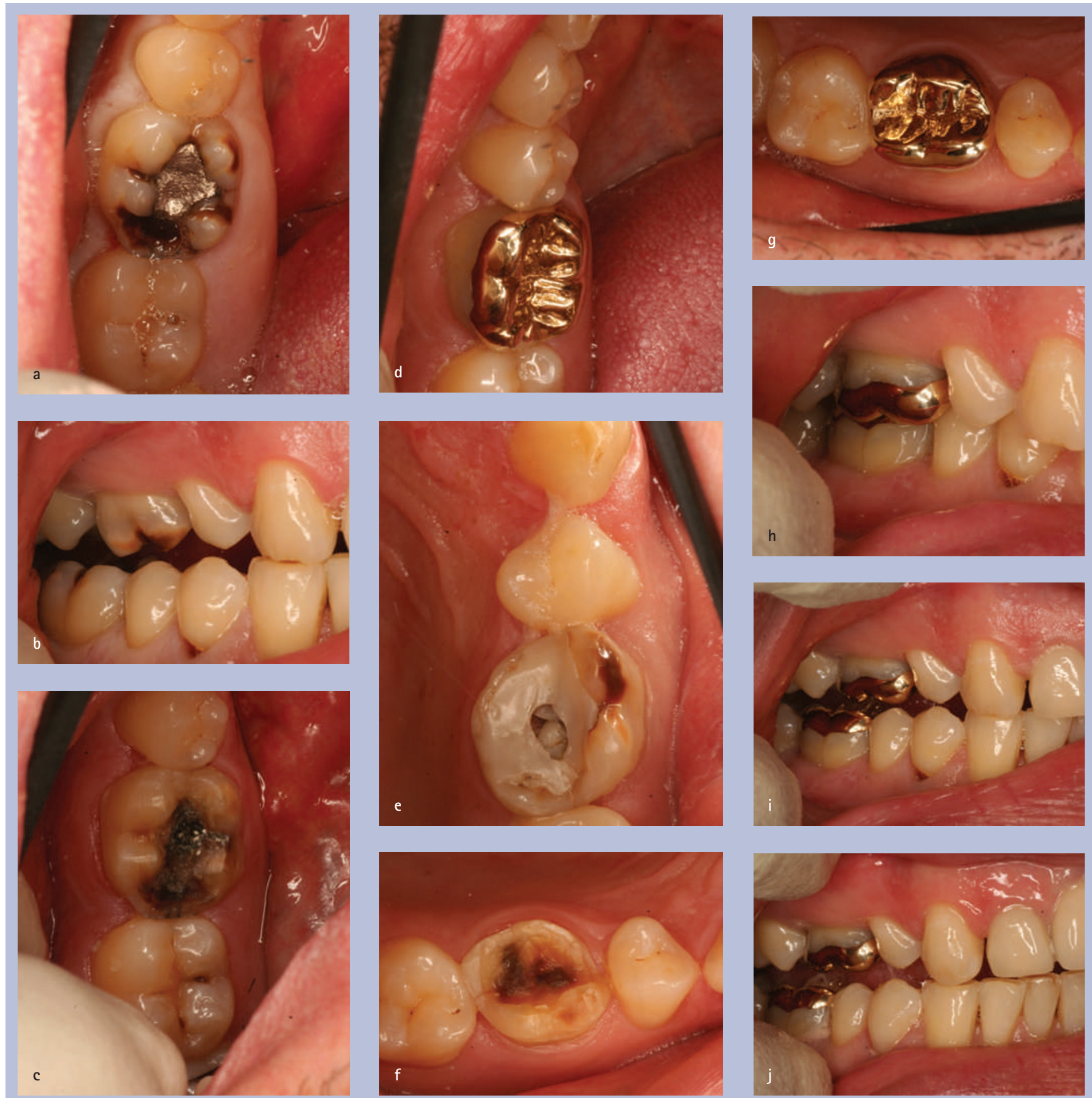

Fig. 7 (a) shows an incompletely fractured symptomatic lower molar tooth, (b) shows the presence of a canine guided occlusion despite marked canine wear. (c) shows that the tooth has been prepared to receive a cast type III gold adhesive onlay with the occlusal surface reduced by 1.0-1.5 $\mathrm{mm}$ and a chamfer margin applied circumferentially. (d) shows the bonded metal onlay restorations in situ. (e) shows the upper molar tooth with a leaking posterior composite restoration and this tooth has also been prepared (f) to receive an adhesive onlay restoration ( $\mathrm{g}$ ) with occlusal contacts shown in (h). Canine guidance is shown in (i) and this was modifed by the addition of resin composite to ensure posterior disclusion upon lateral excursive movements, to provide protection to the recently placed restorations (j)

offers the advantage of being biologically conservative of tooth tissue. Additionally, it would also be reasonable to presume that trauma to the pulpal tissues (which would result from the process of tooth preparation) would be less likely from the effects of less invasive tooth preparations (than would be the case from more aggressive tooth reductions). It has been well documented that the prognosis of endodontically treated, fractured teeth is relatively poor. ${ }^{35}$

The application of provisional adhesive metal onlay restorations may, however, be challenging, as may be the presence of close proximal contact points, where it may be necessary to extend the preparation beneath the contact area. ${ }^{47}$ The preparation and placement of metal adhesive onlay restorations is also economically costly and time consuming. The use of adhesive metal onlays is also contra-indicated among patients who display signs of poor motivation and plaque control respectively, where there are high aesthetic demands and where there is the poor availability of tooth enamel at the restorative margins. ${ }^{47}$ 
The placement of metal adhesive onlays in supra-occlusion may be a possible variation on the approach adopted by Chana et al. ${ }^{46}$ permitting a possible "ultra-conservative approach' to treat CTS (utilising the well documented Dahl concept), although it is imperative that a positive diagnosis is derived, and careful case selection undertaken where the placement of a restoration in supra-occlusion has been planned.

Figure 2 illustrates a case where a gold adhesive onlay has been placed initially in supra-occlusion for a tooth which has been affected by an incomplete fracture. The occlusal contacts have re-established by the three month review. This is in agreement with studies where crowns are intentionally fitted high to avoid the need for occlusal reduction. ${ }^{10}$

\section{Ceramic onlays (conventional/CAD-CAM)}

The ability to etch and bond ceramic materials to tooth enamel offers a possible aesthetic alternative to the use of metal onlays. With the advent of more recent leucite reinforced ceramics it has been possible to fabricate all porcelain restorations with a higher fracture resistance and increased flexural and compressive strengths respectively. ${ }^{2}$

Liebenberg $^{2}$ has published a report that describes the protocol for the fabrication of 'partial coverage porcelain onlays' to treat cases of cracked, painful teeth. The use of such restorations is claimed to be very effective in the treatment of CTS based on anecdotal evidence of treating several cases over a period of at least five years. ${ }^{2}$

Tooth preparation to receive the above form of restoration essentially involves the reduction of the weakened cup by 2.0 $\mathrm{mm}$, followed by the placement of a chamfer/rounded shoulder of $2 \mathrm{~mm}$ in width applied just beneath the prepared occlusal surface(s). ${ }^{2}$ Emphasis has been placed on the presence of enamel around the entire peripheral margin and accurate preparation/impression making.

The use of ceramic onlays clearly involves a greater level of tooth reduction than that advocated for the placement of metal adhesive onlays. As discussed above, this would culminate in a preparation approach that is less biologically conservative than that for the preparation for an adhesive metal onlay, yet less invasive than that for a full coverage restoration. The application of an indirect ceramic would also necessitate the use of a provisional restoration. The need for a provisional restoration in cases of CTS has been postulated to increase the risk of pulpal complications. ${ }^{25}$

However, with the advent of contemporary computer aided design/computer aided manufacturing (CAD/CAM) systems, such as Cerec (Sirona, USA), it is possible to complete a restoration in one visit. The advantages of a CAD/CAM porcelain partial onlay restorations in cases of CTS, in particular where acute thermal symptoms are present according to Griffin ${ }^{1}$ include, the absence of irritation from temporary fabrication and cementation, reduced risk of salivary and bacterial ingress from less than ideal provisional restorations (which may show signs of fracture, leakage or displacement) and the absence of pulpal stresses from a second visit which would include restoration 'try-in', cementation, further tooth desiccation and bacterial exposure or possible hand-piece trauma.

A report has been published describing two cases of CTS which were successfully managed for a period of at least two years post restoration by the application of CAD/ CAM generated partial coverage bonded porcelain onlays. ${ }^{1}$ It is important to note though that many practitioners may not have ready access to $\mathrm{CAD} / \mathrm{CAM}$ facilities; furthermore the inability to use 'layering techniques' culminates in restorations which may not carry optimal aesthetic value. Technique sensitivity is a feature commonly seen as a drawback to the use of CAD/CAM manufacturing systems.

While dental porcelains can be used to produce restorations of a high aesthetic value, their property of low critical strain in tension culminates in a relatively brittle material which may display signs of fracture with little or no plastic deformation. Processing flaws within the material substructure may also lead to eventual cracking. Occlusal adjustments of ceramic restorations may initiate micro-fractures, render the exposure of unglazed ceramic (which may cause wear of the opposing dentition) and repair of ceramic restorations may be challenging.

Porcelain bonded partial coverage onlays offer a more conservative alternative to full coverage restorations for the management of CTS, with high aesthetic value. However, their application is time consuming (even with single visit restorations) and costly.

Of particular concern to the present authors is the high biological cost in terms of occlusal surface reduction, which may be concomitantly associated with a higher risk of irreversible pulpal damage (than may be the case with a metal adhesive onlay preparation). The need to undertake endodontic treatment may not only compromise the longevity of the affected tooth, but also require re-making of the ceramic onlay prosthesis. The latter is not only economically costly, but will also involve the further loss of healthy tooth tissue.

\section{Indirect bonded resin composite onlays}

The bonded indirect composite onlay restoration offers an alternative treatment option to the use of either direct composite onlays or ceramic onlays in the treatment of CTS. Indirect composite onlay restorations have been shown to be effective for the treatment of painful, cracked teeth. ${ }^{49}$

Depicted by Figure 8 is an example of a symptomatic fractured, vital posterior tooth which has been restored by the means of an indirect resin composite onlay. Note the need for a more aggressive level of tooth reduction, when compared to the preparation for a tooth to receive a cast adhesive gold onlay, as shown by Figure 7 .

Deliperi et $a l .{ }^{50}$ state that in comparison to direct composite resin onlays, the indirect technique allows for restorations which have more favourable anatomical form, a more accurate occlusal prescription and more predictable proximal contact anatomy. It has been well documented that the feature of polymerisation shrinkage is a major drawback associated with the use of direct composite restorations. The latter complication is largely negated with the use of indirect techniques (as much of this takes place extra-orally); however, polymerisation shrinkage occurring at the level of the resin lute may still feature. According to Wendt, ${ }^{51}$ on account of a higher level of polymerisation conversation attainable with indirect methods, such restorations can be expected to display improved mechanical and physical properties such as strength and wear resistance.

When compared to bonded porcelain onlays, both form of restoration require the 
same level of tooth reduction. However, indirectly bonded resin composite onlay restorations offer the merits of ease of repair (with direct materials) and adjustment respectively. They are also less abrasive towards opposing occlusal surfaces. The potential to add direct composite may be crucial in cases of CTS, where loss of vitality may precede the cementation of the definitive restoration, as the latter can be readily repaired post-endodontic therapy, without the need for a remake. Furthermore, the indirect composite onlay will continue to serve its purpose as a splint, while root canal therapy is being undertaken (particularly in cases where treatment may be more than one visit).

Indirect composite onlays have been shown to display a greater capacity to absorb compressive loading forces, thereby reducing the impact force on the underlying tooth structure by $57 \%$, when compared to dental porcelain. The latter property is thought to be accounted for by the lower elastic modulus presented by composite resin. A reduction in the load transmitted to a fractured flexible tooth is critical in preventing further progress of the crack. Brunton et al. ${ }^{49}$ showed that premolar teeth restored by the means of composite onlays exhibited higher levels of fracture resistance than equivalent teeth restored with ceramic onlays.

It would appear that the weak link associated with the use of indirect resin composite restorations is the resin based lute. ${ }^{50}$

Signore et al. ${ }^{52}$ have published the results of a six year retrospective study describing the clinical performance of bonded indirect composite onlays for the treatment of 43 cracked, painful teeth. For cases where cavity widths were less than half the distance from the central fossa to the cusp tip, only the involved cusp was reduced; for larger cavities, complete cuspal coverage was undertaken. All cavities were first pre-restored with a directly bonded resin composite, followed by a reduction by 1.5 $2.0 \mathrm{~mm}$ of the occlusal surface; all margins were placed on sound enamel and usually placed supra-ginigivally. Onlays were cemented using a 3-step total etch system with the use of a dual cured low viscosity composite cement (Variolink, Ivoclar, Vivadent). A favourable survival rate of 93.02\% was reported, suggesting that this form of restoration may represent a successful method of treating incompletely fractured posterior teeth.

Indirect resin composite onlays appear to have a role in the management of CTS. They can provide an effective treatment option and can overcome many of the drawbacks associated with the use of directly bonded composite onlays. While their placement requires more tooth preparation, they are more conservative than full coverage restorations and offer the potential for effective repair. Cost and time factors, together with the need for temporisation, are obvious disadvantages. Caution may need to be applied when considering their placement among patients who display signs of tooth wear. ${ }^{40}$

\section{Full coverage crowns}

Based on their observation that cracks on posterior teeth typically extend from the occlusal incline to the cervical third of the clinical crown, Gutherie et al. ${ }^{8}$ have advocated the use of full coverage crowns to be the most appropriate form of restoration to manage cases of cracked tooth syndrome. It has been argued that the resistance form provided by a full coverage restoration enables occlusal forces to be distributed over the entire prepared tooth, thereby minimising stresses which would otherwise be relayed to the crack; while the retention form of the crown through the process of frictional contact and by the action of the cement lute helps to splint the tooth fragments, thereby minimising their independent movement when occlusal forces are released. ${ }^{8}$

A modified preparation to the conventional full coverage preparation (for either a full veneer preparation or that for a metallo-ceramic crown) has been described, which is thought to reduce the functional stresses applied to the fractured segments..$^{56}$ These modifications include: a further reduction of the involved cusp with the application of a subsequent bevel, the avoidance of the placement of boxes and grooves on or adjacent to the fractured aspects of the tooth, placement of margins more apically (which will improve bracing of the crown by the surrounding tooth), the use of adhesive core materials, the use of thicker die spacers to permit the fabrication of a passively fitting restorations and the use of low viscosity cements.

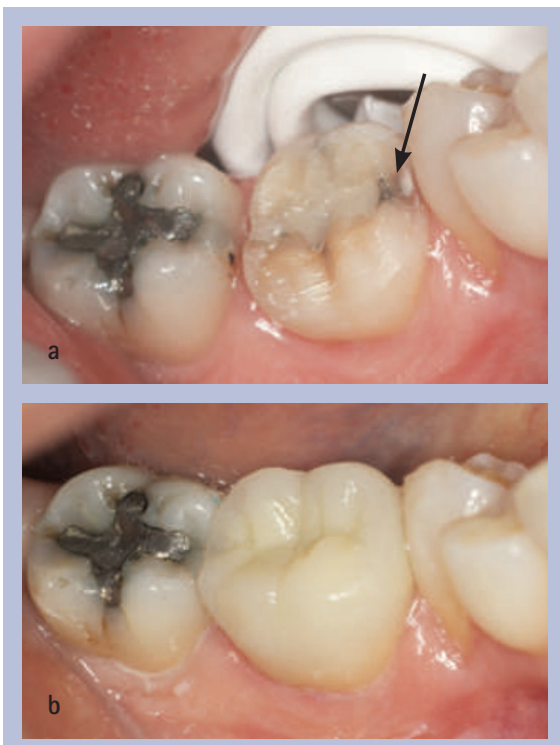

Fig. 8 An example of a symptomatic fractured, vital posterior tooth (a) with a mesial fracture (see arrow) which has been restored by the means of an indirect resin composite onlay (b)

The efficacy of full coverage acrylic provisional crowns in the management of cases suffering from CTS was evaluated by Gutherie et $a l .^{8}$ A failure rate of $11 \%$ was reported; all of the failures required endodontic therapy.

In a more extensive study, where 127 cases of posterior teeth with cracks with concomitant symptoms of reversible pulpitis were restored with full coverage definitive crowns, a staggering 21\% of cases were reported failed within the first six months of evaluation; all cases required subsequent root canal therapy. ${ }^{53}$ Cases with single marginal ridge fractures (either mesial or distal) were reported to be more likely to be in need of endodontic treatment. The proportion of teeth (with CTS) with the subsequent need of further endodontic therapy ${ }^{8,53}$ is significantly higher following the application of full coverage crowns than among those cases which have been splinted by other restorative means as described above.

Various studies have reported the loss of vitality following the application of full coverage single unit crowns to be in the range of 15 to $19 \% .^{54,55}$ Loss of pulpal vitality is an obvious problem following the preparation of teeth to receive the latter form of restoration; the problem appears to be further compounded in cases of CTS, where reversible pulpitis would generally be an already pre-existing condition. It has 
been reported by Tan et al. ${ }^{35}$ that endodontically treated, cracked teeth have a relatively poor long term outlook.

In cases where root canal treatment is indicated following the application of a definitive crown, this may necessitate the remaking of the crown which further adds to the cost of the procedure. The preparation of teeth to receive full coverage crowns is also time consuming.

\section{SUMMARY}

A number of definitive different restorations/restorative techniques have been described for their respective use to treat posterior teeth affected by CTS. However, there is only very limited clinical evidence available in the dental literature to substantiate the use of any of them.

The prognosis of a tooth affected by CTS is determined by three principal factors, hence: the extent and location of the fracture, the point in time when restorative intervention is initiated and thirdly by the type of restoration applied to splint the fracture.

The prognosis of teeth involved by CTS, where the presenting cavity is not in excess of one half of the bucco-linigual width when restored by the means of directly bonded plastic restorations without cuspal coverage, appears to be reasonably acceptable. The preparation of teeth to receive such restorations is biologically conservative, with the subsequent loss of pulp vitality not being an associated primary concern. The prognosis of root filled cracked teeth in the longer term appears poor. ${ }^{35}$

According to a leading authority, teeth affected by CTS should be initially restored by means of bonded direct restorations as a means of immediate/intermediate therapy; where an indirect restoration is indicated from the outset, such a restoration should only be placed after having managed the immediate symptoms. ${ }^{25,26}$

Conventional full coverage crowns and conventional onlays have been utilised as a historical gold standard for the above purpose. ${ }^{57}$ With the former, the copious loss of tooth tissue and high risks of loss of pulp vitality respectively are major concerns, which may impart a considerable negative effect on the prognostic outcome of teeth affected when treated by such an approach. The need for provisional restorations, cost and time factors are also obvious drawbacks to the use of conventional indirect restorations.

Adhesive metal and indirect resin composite onlays may in time show some level of considerable promise for improving the prognosis of teeth affected by CTS, and form excellent definitive, long term restorative options for the treatment of teeth affected by this condition, in particular where affected teeth may have been intruded by ultra-conservative restorations placed in supra-occlusion. A more biologically conservative tooth preparation will without doubt help to prolong the longevity of the tooth but also help to prevent irreversible pulpal damage.

Of the limited data available, it would be fair to conclude that direct restorations with cuspal coverage, in particular bonded composite restorations appear to be the most beneficial when considering prognostic outcome of teeth restored for the purposes of incomplete posterior tooth fractures..$^{25,26}$ Furthermore, their application is cost effective, efficient and they also permit an aesthetic outcome with limited damage inflicted upon the dental pulp. ${ }^{26}$ However, the placement of direct composite onlay restorations is very dependent upon operator skill, and furthermore, some level of tooth preparation is still required.

1. Griffin J. Efficient, conservative treatment of symptomatic cracked teeth. Compendium 2006; 27: 93-102.

2. Liebenberg W H. Partial coverage indirect tooth coloured restorations; steps to clinical success. Am J Dent 1999; 12: 201-209.

3. Agar J R, Weller R N. Occlusal adjustments for initial treatment and prevention of cracked tooth syndrome. J Prosthet Dent 1988; 60: 145-147.

4. Hiatt W H. Incomplete crown-root fractures in pulpal periodontal disease. J Periodontol 1973; 44: 369-379

5. Fox K, Youngson C C. Diagnosis and treatment of the cracked tooth. Prim Dental Care 1997; 4: 109-113.

6. Geurtsen W. The cracked tooth syndrome; clinical features and case reports. Int J Periodontics Restorative Dent 1992; 12: 395-405.

7. Ehrmann E H, Tyas M J. Cracked tooth syndrome: diagnosis, treatment and correlation between symptoms and post-extraction findings. Aust Dent $J$ 1990; 35: 105-112.

8. Gutherie G C, Difiore P M. Treating the cracked tooth with a full crown. J Am Dent Assoc 1991; 122: 71-73.

9. Dahl B, Krogstad O, Karlsen K. An alternative treatment of cases with localised attrition. J Oral Rehabil $1975 ; 2: 209-214$

10. Hemmings K W, Darbar U R, Vaughan S. Tooth wea treated with direct composite restorations at an increased vertical dimension: results at 30 months. J Prosthet Dent 2000; 83: 287-293.

11. Gerasimidou 0. Tooth mobility, movement and symptoms caused by occlusal changes. PhD Thesis. King's College London, 2009.

12. Poyser N J, Porter R W J, Briggs P F A, Channa H S, Kelleher M G D. The Dahl concept: past, present and future. Br Dent J 2005; 198: 669-676

13. Geurtsen W, Garcia-Godov F. Bonded restorations for the prevention and treatment of the cracked tooth syndrome. Am J Dent 1999; 11: 266-270.

14. Plasmans P J, Breugers N H, Mulder J. Long term survival of extensive amalgam restorations. J Dent Res 1998; 77: 453-460.

15. Van Nieuwenhuysen J P, D'Hoore W, Carvahlo J, Quist V. Long term evaluation of extensive restorations in permanent teeth. J Dent 2003; 131: 395-405.

16. Staninec M, Holt M. Bonding of amalgam to tooth structure: tensile adhesion and microleakage tests. J Prosthet Dent 1988; 59: 397-402.

17. Trushkowsky R. Restoration of a cracked tooth with a bonded amalgam. Quintessence Int 1991; 22: 397-400.

18. Ueno Y. A clinical evaluation of adhesive amalgam lining with $4 \mathrm{META} / \mathrm{MMA} \mathrm{TBB}$ adhesive resin without anaesthesia. J Jpn Soc Adhes Dent 1989: 73: 181-189.

19. Bearn D, Saunders E, Saunders W. The bonded amalgam restoration - a review of the literature and report of its use in the treatment of four cases of cracked tooth syndrome. Quintessence Int 1994; 25: 321-326.

20. Omura I, Yamauchi J, Harada I, Wada T. Adhesive and mechanical properties of a new dental adhesive. J Dent Res 1984; 62: 233.

21. Eakle W S, Staininec M, Lacy A M. Effect of bonded amalgam on the fracture of teeth. J Prosthet Dent 1992; 68: 257-260.

22. Oliveira J P, Cochran M A, Moore B K. Influence of bonded amalgam restorations on the fracture strength of teeth. Oper Dent 1996; 21: 110-115.

23. Geurtsen W, Orth M, Gartner A. Fracture resistance of human maxillary molars with MOD amalgam or composite fillings. Dtsch Zahnarztl Z 1989; 44: 108-110.

24. Ausiello P, DeGee A S, Rengo S et al. Fracture resistance of endodontically treated premolars adhesively restored. Am J Dent 1997; 10: 237-241.

25. Opdam N J, Roeters J J. The effectiveness of bonded composite restorations in the treatment of painful, racked teeth: six month evaluation. Oper Dent 2003 28: 327-333.

26. Opdam N J, Roeters J J, Loomans R A, Bronkhorst E. Seven year clinical evaluation of painful, cracked teeth restored with a direct composite restoration. J Endod 2008; 34: 808-811.

27. Weiczkowski G, Joynt R B, Klockowski R et al. Effects of incremental versus bulk fill technique on resistance to cuspal fractures of teeth restored with posterior composites. J Prosthet Dent 1988; 60: 283-288.

28. Van Dijken J W V. Direct resin composite inlays/ onlays: an 11 year follow up. J Dent 2000; 28: 299-300.

29. Stavridakis M M, Kakaboura A I, Ardu S, Krejci l. Marginal and internal adaptation of bulk filled class I and cuspal coverage direct resin composite restorations. Oper Dent 2007; 32: 515-523.

30. Hoffman W, Just N, Haller B, Hugo B, Klaiber B. The effect of glass ionomer cements or composite resin bases on restoration of cuspal stiffness of endodntically treated premolars in vitro. Clin Oral Investig 1998; 2: 77-83.

31. McCullock A S, Smith B G N. In vitro studies of cuspal reinforcement with adhesive restorative materials. Br Dent J 1986; 161: 450-452.

32. Krejci I, Lutz F, Krejci D. The influence of different base materials on marginal adaptation and wear of conventional class II composite resin restorations. Quintessence Int 1988; 19: 191-198.

33. Roulet J F. Benefits and disadvantages of tooth coloured alternatives to amalgam. J Dent 1997; 25: 459-473.

34. Gutmann J L, Rakusin H. Endodontic and restorative management of incompletely fractured molar teeth. Int Endod J 1994; 27: 343-348.

35. Tan I, Chen N N, Poon CY, Wong H B. Survival of root filled cracked teeth in a tertiary institution. Int Endod J 2006; 39: 886-889.

36. Hodd J A A. Biomechanics of the intact, prepared and restored tooth; some clinical implications. Int Dent J 1991; 41: 25-32 
37. Homewood C I. Cracked tooth syndrome - incidence, clinical findings and treatment. Aust Dent J 1998; 43: 217-222.

38. Davis R, Overton J. Efficacy of bonded and nonbonded amalgams in the treatment of teeth with incomplete fractures. J Am Dent Assoc 2000; 131: 496-478.

39. Fennis W M, Kuijs R H, Kreulen C M, Verdonschot $N$, Creugers N H. Fatigue resistance of teeth restored with cuspal coverage composite restorations. Int J Prosthodont 2004; 17: 313-317.

40. Bartlett D, Sundaram G. An up to 3 year randomized clinical study comparing indirect and direct resin composite used to restore worn posterior teeth. Int J Prosthodont 2006; 19: 613.

41. Shillingburg $H T$, Hobo $S H$, Lowell D W, Jacobi $R$, Brackett S. Fundamentals of fixed prosthodontics, 3rd ed. pp 171-180. Quintessence Publishing, 1997.

42. Roznowski M, Bremer B, Geurtsen W. Fracture resistance of human molars restored with various filling materials. In Moermann W H. Proceedings of the international symposium on computer restorations. pp 559-566. Chicago: Quintessence, 1991.

43. Wassell R W, Walls A W G, McCabe J F. Direct composite inlays versus conventional composite restorations: 5 year follow up. J Dent 2000; 28: 375-382.

44. Rosenstiel S F, Land M F, Fujimoto J. Contemporary fixed prosthodontics, 3rd ed. pp 230-261. Mosby, 2001.

45. Behle C A. Conservative direct and indirect resin posterior restorative alternatives for racked dentition. Pract Periodontics Aesthet Dent 1997; 9: 405-413.

46. Chana $H$, Kelleher M, Briggs $P$, Hopper R. Clinical evaluation of resin bonded gold alloys. J Prosthet Dent 2000; 83: 294-300

47. Yap A U J. Cuspal coverage with resin bonded metal onlays. Dent Update 1995; 22: 403-406.

48. Tanaka T, Atusuta M, Nakabayashi M, Masuhara E. Surface treatment of gold alloys for adhesion. J Prosthet Dent 1988; 60: 271-279.

49. Brunton P A, Cattell P, Burke F J T, Wilson N H F. Fracture resistance of teeth restored with onlays of three contemporary tooth-coloured resinbonded restorative materials. J Prosthet Dent 1999; 82: 167-171.

50. Deliperi S, Bardwell D N. Direct cuspal coverage posterior resin composite restorations: a case report. Oper Dent 2009; 30: 143-150

51. Wendt $S L$ Jr. The effect of heat used as secondary cure upon the physical properties of three composite resins. II. Wear, hardness and color stability. Quintessence Int 1987; 18: 351-356.

52. Signore A, Benedicenti S, Covani U. Ravera G. A 4 to 6 year retrospective clinical study of cracked teeth restored with bonded indirect resin composite onlays. Int J Prosthodont 2007; 20: 609-616.

53. Krell K, Rivera E. A six year evaluation of cracked teeth diagnosed with reversible pulptitis: treatment and prognosis. J Endod 2007; 33: 1405-1407.

54. Saunders W P, Saunders E M. Prevalence of periradicular periodontitis associated with crowned teeth in an adult Scottish subpopulation. Br Dent J 1988; 185: 137-140.

55. Cheung G S, Lia S C, Ng R P. Fate of vital pulps beneath a metal ceramic crown or a bridge retainer. Int Endod J 2005; 38: 521-530.

56. Casciari B J. Altered preparation design for cracked teeth. J Am Dent Assoc 1991; 130: 571-572.

57. Christensen G J. The cracked tooth syndrome: a pragmatic treatment approach. J Am Dent Assoc 1998; 124: 107-108. 